\title{
Circadian and Photic Regulation of Phosphorylation of ERK1/2 and Elk-1 in the Suprachiasmatic Nuclei of the Syrian Hamster
}

\author{
Andrew N. Coogan and Hugh D. Piggins \\ School of Biological Sciences, University of Manchester, Manchester, United Kingdom M13 9PT
}

\begin{abstract}
In this study we investigated the circadian and photic regulation of phosphorylation of the extracellular signal-related kinase (ERK) 1/2, and the transcription factor Elk-1 in the suprachiasmatic nuclei of the Syrian hamster. We report that levels of phosphorylated ERK (P-ERK) are rhythmic, peaking during the mid subjective day, whereas phosphorylated Elk-1 (P-Elk-1) shows no distinct rhythm. Light pulses during the subjective night rapidly, but transiently, induce P-ERK, whereas P-Elk-1 is also induced, albeit with a slower time course. Application of the ERK pathway inhibitor U0126 attenuates photic induction of both P-ERK and P-Elk-1 and phase advances of wheel-running behavior. The NMDA receptor channel blocker, MK-801, also significantly attenuates photic induction of P-ERK and P-Elk-1. Taken together, these results indicate a role of the ERK cascade in the regulation of free-running circadian rhythms and of photic-resetting of these rhythms and suggest that in the mammalian suprachiasmatic nuclei, Elk-1 represents a novel molecular component of the photic-induction pathway.
\end{abstract}

Key words: circadian; MAP kinase; clocks; brain; phosphorylation; Elk-1

\section{Introduction}

In mammals the master circadian pacemaker has been localized to the suprachiasmatic nuclei (SCN) of the hypothalamus (Ralph et al., 1990). The SCN clock is reset by daily exposure to light by transmission of photic information from the retina via the retinohypothalamic tract (RHT) (Moore and Lenn, 1972), a monosynaptic glutamatergic projection (Ebling, 1996). The biochemical basis for circadian rhythmicity consists of interlocking feedback/feedforward loops of clock genes and their protein products (Allada et al., 2001; Reppert and Weaver, 2002). The cycling of these factors, and the ensuing clock output generated, is under strong post-translational control, via phosphorylation/ dephosphorylation mechanisms (Lowrey et al., 2000; Lee et al., 2001). However, our understanding of the pathways underlying such regulation is incomplete.

A candidate pathway for transmission of photic information to the core nuclear clockworks is the extracellular signalregulated kinase 1/2 (ERK) pathway. ERKs are members of the mitogen-activated protein (MAP) kinase superfamily that become active after their own phosphorylation on threonine/tyrosine residues by a MAP kinase kinase (Sweatt, 2001). ERKs are also involved in a number of physiological effects that are dependent on glutamatergic neurotransmission (Coogan et al., 1999). Recently, phosphorylated ERK (P-ERK) has been shown to interact directly with clock gene products (e.g., BMAL1) (Sanada et al., 2002) and may also alter the activity of transcription factors and subsequently clock gene expression [e.g., cAMP regulatory ele-

\footnotetext{
Received Dec. 3, 2002; revised Jan. 17, 2003; accepted Jan. 22, 2003.

This work was supported by the Biotechnology and Biological Sciences Research Council and the University of Manchester. We also acknowledge the contribution of Lyle Freeman at the onset of this study.

Correspondence should be addressed to Hugh D. Piggins, 3.614 Stopford Building, School of Biological Sciences, University of Manchester, 0xford Road, Manchester, UK M13 9PT. E-mail: hugh.piggins@man.ac.uk. Copyright $\odot 2003$ Society for Neuroscience $\quad 0270-6474 / 03 / 233085-09 \$ 15.00 / 0$
}

ment binding protein (CREB) regulation of per1] (Obrietan et al., 1999; Oh-Hashi et al., 2002; Tischkau et al., 2002). The ERK cascade has also been implicated in the circadian production of arginine vasopressin (AVP) mRNA by neurons of the SCN (Arima et al., 2002). In the circadian clock, light induces P-ERK in the mouse $\mathrm{SCN}$ in a phase-dependent manner (Obrietan et al., 1998). P-ERK also exhibits a circadian variation in the mouse SCN (Obrietan et al., 1998) and in chick retina (Ko et al., 2001) and pineal gland (Sanada et al., 2000; Hayashi et al., 2001).

A number of putative substrates for the ERK pathway may play a role in circadian processes. One of these is the ternary complex factor Elk-1, a member of the Ets family of transcription factors that after phosphorylation strongly upregulate transcription mediated via serum response elements (SREs) on gene promoter sequences. In brain, such events can be induced via glutamatergic receptor activation (Vanhoutte et al., 1999) and can be blocked by inhibitors of the ERK pathway (Davis et al., 2000). A number of photically induced genes in the SCN [e.g., c-fos (Rusak et al., 1990) and perl (Wilsbacher et al., 2002)] have SREs on their promoter sequences, raising the possibility that the ERK pathway may transduce its effects by transcriptional regulation via Elk-1. Thus Elk-1 may represent a novel input point to the core molecular clockworks. To this end we have examined circadian and photic regulation of ERK and Elk-1 in the Syrian hamster SCN and have found strong evidence of a role for ERK/Elk-1 in determining phase in the SCN clock.

\section{Materials and Methods}

Animals. All procedures were performed in accordance with the UK Home Office Animals (Scientific Procedures) Act 1986 by appropriately qualified staff. Adult male Syrian hamsters (120-150 gm; LVG strain; Charles River, Margate, Kent, UK) either were housed in groups of six for diurnal experiments or were housed singly in cages equipped with running wheels for determination of circadian phase using the Stanford 
Chronobiology Kit PC software (Stanford Software Systems, Santa Cruz, CA). Animals were housed either under light/dark (LD) conditions (14 hr light/10 hr dark; 80 lux light) or in constant dark (DD) for either two cycles (light-pulse experiments) or seven cycles (free-run experiments). Under diurnal conditions, Zeitgeber time (ZT) 0 is defined as the start of the lights-on phase, whereas under free-running conditions circadian time (CT) 12 is defined as the clear onset of wheel running for each individual hamster. For all experiments, food and water were provided ad libitum.

Tissue preparation. Across both the LD and the DD cycles, animals were anesthetized with sodium pentobarbitone (Pentoject, Animalcare, York, UK; $120 \mathrm{mg}$ ) and perfused intracardially first with $0.9 \%$ saline (50 $\mathrm{ml}$ ) followed by $100-150 \mathrm{ml}$ of Zamboni's modified fixative (4\% paraformaldehyde, $15 \%$ picric acid in $0.1 \mathrm{~m}$ phosphate buffer, $\mathrm{pH} 7.35)$. The brains were subsequently removed from the skull case and postfixed in Zamboni's modified fixative overnight at $4^{\circ} \mathrm{C}$ before being cryoprotected in $30 \%$ sucrose solution for a further $48 \mathrm{hr}$. Brains were then frozen on dry ice and cut into $30-\mu \mathrm{m}$-thick coronal sections using a freezing stage sledge microtome (Brights Instruments, Huntingdon, UK).

Photic regulation. For light-pulse experiments, animals were maintained under an LD cycle before being released into DD for two cycles. During the third cycle the lights ( $~ 80$ lux $)$ were switched on for $30 \mathrm{~min}$, starting at CT8, CT13, or CT18. For light pulses at CT18, animals were sampled at CT17 and CT17.5 (before pulse), at CT18.1 and CT18.5 (during the light pulse), and at CT19 and CT19.5 (after the end of the light pulse). In one series of experiments the lights were left on for the duration of the sampling period (i.e., CT18-CT19.5). For light pulses at CT8 and CT13, animals were sampled during the preceding hour and from 15 to $70 \mathrm{~min}$ after the start of the pulse. Given the time course of induction of P-ERK, P-Elk-1, and c-Fos (see Figs. 1, 2, 5), only animals from appro- priate time points after the pulse were used for analysis (i.e., P-ERK was assessed at 15-45 min after the onset of the pulse).

Immunohistochemistry. Free-floating sections were washed two times for $10 \mathrm{~min}$ in $0.1 \mathrm{M}$ phosphate buffer (PB) followed by one wash for 10 $\min$ in $\mathrm{PB}+0.03 \%$ Triton X-100 (PBX). Sections were then incubated in $1.5 \%$ hydrogen peroxide in $\mathrm{PB}$ for 20 min followed by another two washes in PB and one in PBX followed by incubation at room temperature for $1 \mathrm{hr}$ in PBX containing 5\% normal goat serum (NGS). Labeling of P-ERK, P-Elk-1, ERK, Elk-1, and c-Fos was performed using the following primary antibodies: P-ERK, mouse monoclonal antibody specific for ERK1 and ERK2 only when dually phosphorylated on Thr-202 and Tyr-204 (dilution 1:500; Cell Signaling Technologies, Hitchin, UK); P-Elk-1, mouse monoclonal antibody recognizing Elk-1 only when phosphorylated at Ser-383 (dilution 1:80; Santa Cruz Biotechnology); ERK, rabbit polyclonal antibody raised against subdomain XI of ERK1, also recognizing ERK2 (dilution 1:150; Santa Cruz Biotechnology); Elk-1, rabbit polyclonal antibody raised against the $\mathrm{C}$ terminus sequence of human Elk-1 (dilution 1:150; Santa Cruz Biotechnology); c-Fos, rabbit polyclonal antibody raised against the amino terminus of human c-Fos (dilution 1:8000; Santa Cruz Biotechnology). All incubations with primary antibodies took place in PBX with $2 \%$ NGS for $36-48 \mathrm{hr}$ at $4^{\circ} \mathrm{C}$. The specificity of the P-Elk-1 antiserum was confirmed in a number of sections from light-pulsed animals that were incubated with P-Elk-1 antibody preadsorbed for $1 \mathrm{hr}$ with P-Elk-1 control peptide (Santa Cruz Biotechnology). After the incubation with primary antibody, sections were again washed in PB and PBX and then incubated with biotinylated secondary antibodies (goat anti-rabbit, Vector Laboratories, Peterborough, UK; goat anti-mouse, Chemicon, Harrow, UK; both 1:400 dilutions) for $80 \mathrm{~min}$. Sections were washed again before incubation with avidin-biotin-peroxidase complex (0.4\%; Vector Laboratories) for 90

A
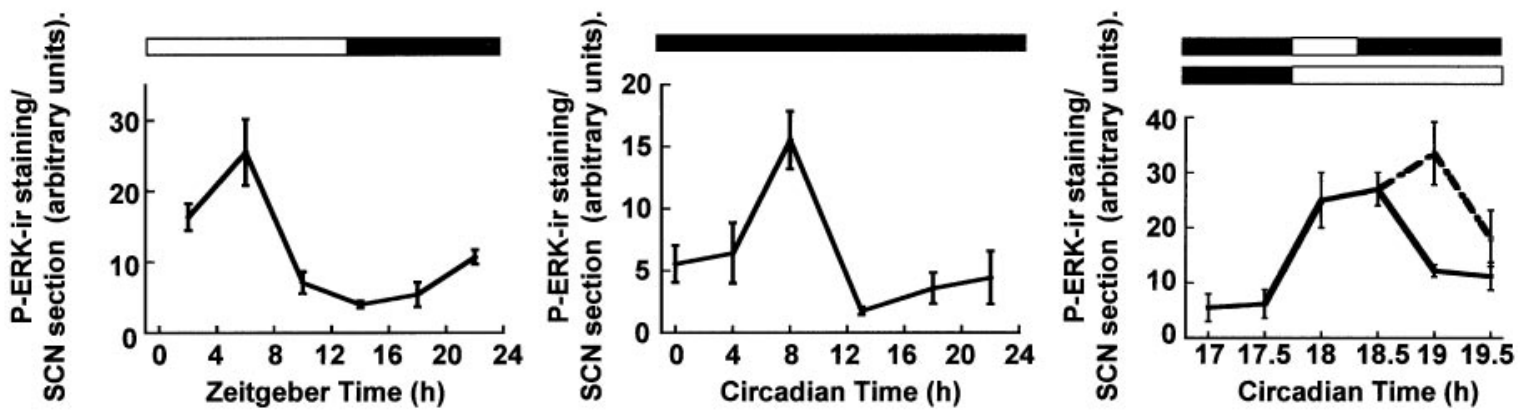

D

CT8



B
C
CT18

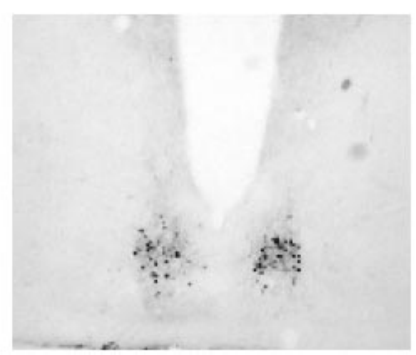

CT18 + Light

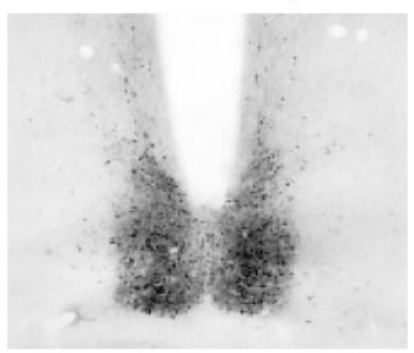

Figure 1. Regulation of P-ERK in the hamster SCN. A, Diurnal variation of P-ERK levels across an LD cycle. $B$, Circadian variation of P-ERK in constant darkness. Under both diurnal and free-running conditions, P-ERK levels peak during the mid subjective day, and levels are low throughout the subjective night. C, Photic regulation of P-ERK in the hamster SCN. Animals were sampled before, during, and after a $30 \mathrm{~min}$ light pulse (CT18-18.5; solid line) and during a $90 \mathrm{~min}$ (CT18-19.5) light pulse (dashed line). Onset of the light pulse triggered a large rise in levels of P-ERK in the SCN that rapidly declined toward baseline on termination of the pulse. Elongation of the light pulse led to sustained P-ERK levels. $n=4-6$ for each point in $A-C$. $D$, Representative photomicrographs of P-ERK staining in the SCN sampled during the subjective day in DD (CT8), during the subjective night in DD (CT18), and 30 min into a light pulse (CT18 + Light). Scale bar, $50 \mu \mathrm{m}$. 
A

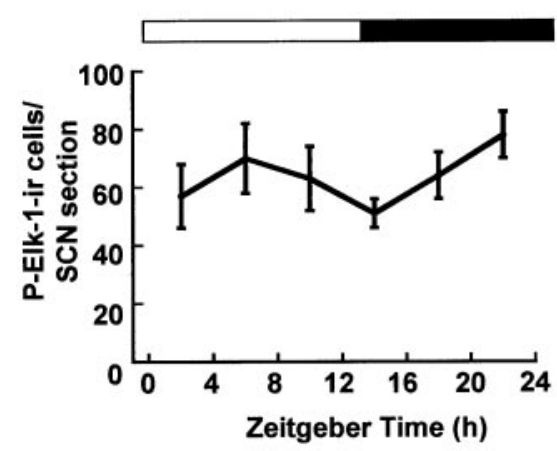

D

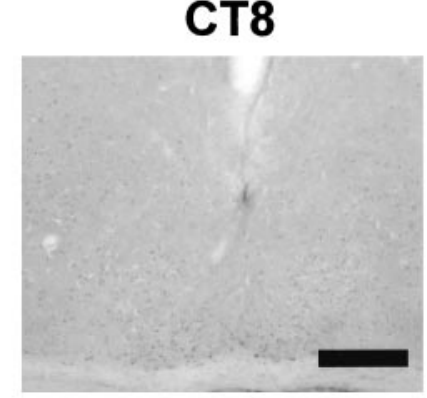

B

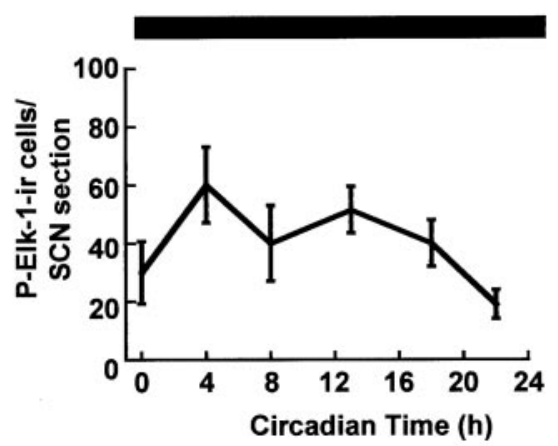

CT18

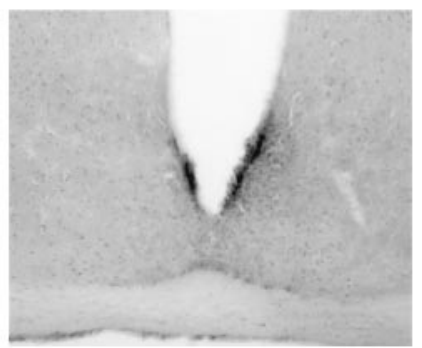

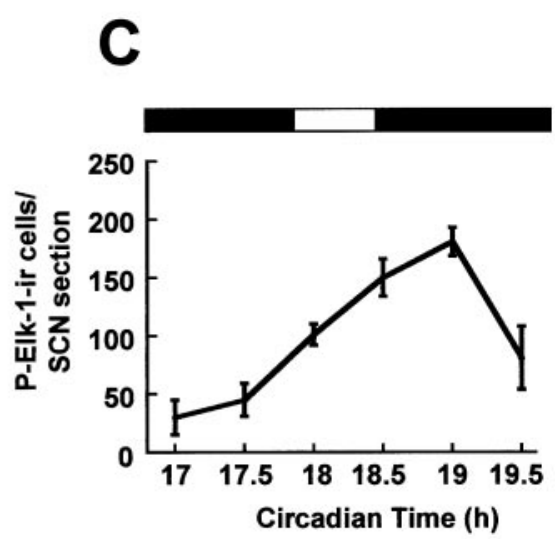

CT18 + Light

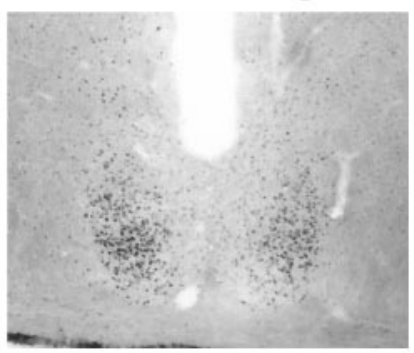

Figure 2. Regulation of P-Elk-1 in the hamster SCN. A, Diurnal variation of P-Elk-1 levels across the LD cycle. B, Circadian variation of P-Elk-1 in constant darkness. Under both diurnal and free-running conditions, P-Elk-1 levels did not vary significantly. C, Photic regulation of P-Elk-1 in the hamster SCN. Animals were sampled before, during, and after a 30 min light pulse (CT18 -18.5; solid line). Application of the light pulse triggered a rise in levels of P-Elk-1 in the SCN, with peak levels achieved 60 min after onset of the pulse. $n=4-6$ for each point in $A-C$. D, Representative photomicrographs of P-Elk-1 staining in the SCN sampled during the subjective day in DD (CT8), during the subjective night in DD (CT18), and 60 min after onset of a light pulse (CT18 + Light). Scale bar, $50 \mu \mathrm{m}$.

min in lightproof conditions at room temperature. Sections were again washed two times for $10 \mathrm{~min}$ in $\mathrm{PB}$ followed by one wash in $0.1 \mathrm{M}$ sodium acetate, $\mathrm{pH}$ 6, followed by visualization of antigen using the nickel DAB method with glucose oxidase (Sigma, Poole, UK) as the catalyst. Sections for each antigen were reacted for standardized lengths of time to allow for uniform intensity of staining across experimental groups. Sections were then mounted onto gelatin-coated slides, dried, dehydrated, cleared in Histoclear (National Diagnostics, Hull, UK), and coverslipped using Entellan (Merck, Dorset, UK).

Surgery. Adult male Syrian hamsters were anesthetized with a ketamine/xylazine mixture (200 mg/kg ketamine; $10.8 \mathrm{mg} / \mathrm{kg}$ xylazine) and mounted into a stereotaxic frame for implantation of guide cannulas for intracerebroventricular microinjection into the third ventricle. Stainless steel guide cannulas ( $22 \mathrm{ga}, 9 \mathrm{~mm}$; Plastics One) were aimed at coordinates of $0.1 \mathrm{~mm}$ lateral from bregma and $7.2 \mathrm{~mm}$ ventral to the skull surface. After the cannula was fixed in place with dental cement, a cannula dummy was fitted obtruding $0.5 \mathrm{~mm}$ beyond the guide. Animals were then treated with the analgesic buprenorphine $(0.05 \mathrm{mg} / \mathrm{kg})$ and allowed to recover for 7-10 $\mathrm{d}$ in $\mathrm{LD}$. After this period animals were placed in DD for two cycles, and on the third cycle they received a microinjection of the MAP kinase kinase (MEK) inhibitor U0126 (Affiniti Research Products, Exeter, UK) $(1,2.5$, or $5 \mathrm{nmol} / 1 \mathrm{ml})$ or vehicle (10\% DMSO in $0.9 \%$ saline) via a 28 gauge, $9.5 \mathrm{~mm}$ injection cannula connected to a $1 \mu \mathrm{l}$ Hamilton microsyringe. Application of drug or vehicle occurred in DD, with the aid of an infrared viewer, $30 \mathrm{~min}$ before application of a CT18 light pulse. After microinjections, the cannula was left in situ for at least 1 min before being withdrawn to prevent backflow. Cannula placement was verified from sections processed for immunohistochemistry. Only animals with the cannula placed in the ventral half of the third ventricle (within $400 \mu \mathrm{M}$ of the dorsal SCN) were included for analysis.

MK-801 treatments. Hamsters were maintained in LD for 14 cycles and then released into DD. On the third cycle in DD animals were injected with MK-801 (5 mg/kg, i.p.; RBI, Poole, UK) or vehicle (10\% DMSO in $0.9 \%$ saline) at CT17.5, and then lights were switched on at CT18. Animals were sampled between CT18.2 and CT19.2 and appropriately processed for P-ERK, P-Elk-1, and c-Fos immunohistochemistry according to the time course of induction of these factors (see Figs. 1, 2, 4).

Behavioral phase-shifting experiments. Cannulated hamsters were maintained in LD in cages equipped with stainless steel running wheels and released into DD for two cycles. On the third cycle animals received an intracerebroventricular microinjection of $\mathrm{U} 0126(5 \mathrm{nmol}$ in $1 \mu \mathrm{l})$ or vehicle ( $1 \mu \mathrm{l}$ of $10 \%$ DMSO in $0.9 \%$ saline) at CT17.5. At CT18 animals received a $30 \mathrm{~min}$ light pulse and were then replaced in DD. Wheelrunning behavior was recorded for another seven cycles after the light pulse, and phase shifts of running wheel behavior were quantitated as the difference between lines plotted through the onset of activity in the cycles before and after the treatment and light pulse, as determined by three individuals blind to the treatments.

Data analysis. Levels of immunostaining and numbers of immunopositive cells for the various antigens were assessed using the Scion Image $\beta$ 4.2 software (Scion Corporation, Frederick, MD). For determination of levels of immunostaining for P-ERK, ERK and Elk-1 images of the SCN region were taken using a digital camera (Camedia C-2020-Z, Olympus) mounted on an Olympus BX-50 microscope. Background levels of staining in the lateral hypothalamus were subtracted, and stain density within a user-defined region of SCN was quantified. For assessing numbers of c-Fos-immunoreactive (ir) and P-Elk-1-ir cells in the SCN, background staining was subtracted, and a thresholding procedure was used to count numbers of stained nuclei. Preliminary studies indicated that manual counts of c-Fos-ir and P-Elk-1-ir nuclei in the SCN closely resembled those obtained using the computerized method, and so only cell counts obtained using Scion Image are reported here. Cell counts and stain 
A

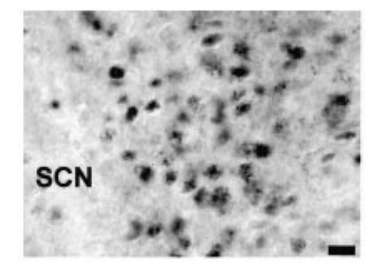

C
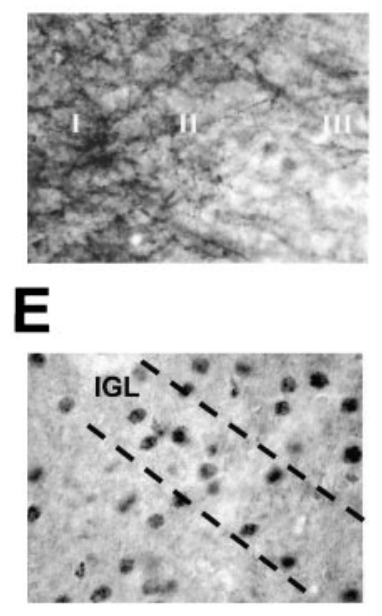

Figure 3. P-Elk-1 staining in other areas of the hamster brain. A, P-Elk-1 staining in the SCN was always found to be nuclear, with no discernable dendritic or axonal staining present (section from CT19, light pulse given (T18-18.5). B, Adjacent section to that shown in $A$, but primary antiserum was adsorbed with phosphorylated Elk-1 peptide. This treatment abolishes P-Elk-1 staining. C, P-Elk-1 staining in the hamster parietal cortex. Here the P-Elk-1 staining is seen to be mostly dendritic and axonal in character, with only faintly stained nuclei present, most notably in layers I, II, and III. D, P-Elk-1 staining in hippocampal CA1. Similar to the cortex, P-Elk-1 staining in the hippocampus is dendritic and axonal in character, present most notably in the stratum radiatum (SR). Weakly stained nucleiare present in the stratum pyramidale(SP).E,P-Elk-1 in the intergeniculate leaflet (IGL). The IGL is part of the geniculate complex and is known to play a part in circadian time keeping in mammals. P-Elk-1-ir was present in the nuclei of IGL cells. P-Elk-1 levels in the IGL were not affected by photic conditions or by circadian phase. Scale bar: (in $A) A-E, 15 \mu \mathrm{m}$.

density were assessed throughout the rostrocaudal extent of the SCN (four to six sections per animal per antigen), and average values per SCN slice were calculated for each animal.

Statistics. Values are reported as mean \pm SEM. For statistical analysis, unpaired two-tailed Student's $t$ tests or one-way ANOVAs with Tukey post hoc test were used on the SigmaStat for Windows PC program (SPSS, Chicago, IL). Significance was set at $p<0.05$.

\section{Results}

\section{Regulation of P-ERK in the hamster SCN}

Levels of P-ERK were assessed in the SCN of Syrian hamsters maintained under an LD cycle, in DD, and in DD given a light pulse at CT18-18.5. Under both diurnal and free-running conditions, P-ERK in the SCN showed significant temporal variation, with levels being high during the subjective day and low during the subjective night (Fig. $1 A, B$ ) (e.g., $p<0.005$ between CT8 and CT18). During the subjective day, P-ERK staining was detected at all rostrocaudal levels of the SCN, with staining present in cell somata, nuclei, and processes. During the subjective night, P-ERK staining was absent in the SCN except for in the mid-caudal SCN, where a cluster of densely stained perikarya was detected consistently (Fig. 1D). Thus P-ERK staining in the SCN displays circadian/diurnal variation not only in the overall levels
A

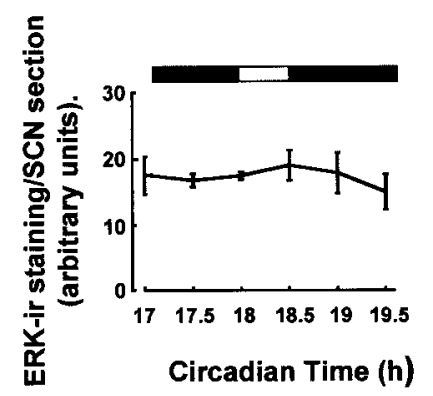

B

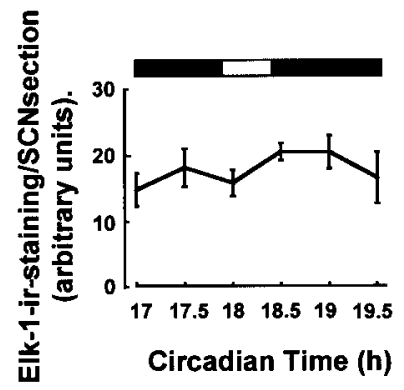

Figure 4. Total levels of ERK and Elk-1 do not vary in the SCN. Total levels of ERK $(A)$ and Elk-1 $(B)$, regardless of phosphorylation state, were not altered by light pulses at CT18. $n=4-6$ for each point in $A$ and $B$. ERK and Elk-1 also did not vary across the circadian or diurnal cycle (data not shown).

of immunostaining, but also in the sub-SCN distribution of that staining.

To examine the photic regulation of P-ERK in the SCN, hamsters received a $30 \mathrm{~min}$ light pulse starting at CT18 [during the phase advance portion of the photic phase-response curve (PRC)]. A time course for regulation of P-ERK staining in the SCN was constructed by sampling animals before $(\mathrm{CT} 17+17.5)$, during $(\mathrm{CT} 18+18.5)$, or after $(\mathrm{CT} 19+19.5)$ the light pulse. On application of the light pulse, levels of P-ERK were found to be rapidly upregulated throughout the SCN $(p<0.005$ between CT17 or 17.5 and CT18 or 18.5) (Fig. 1C). Levels of total ERK protein were unaffected by the light pulse (see Fig. $4 A$ ). After termination of the light pulse, levels of P-ERK began to return to baseline value ( $p<0.05$ between CT18 or 18.5 and CT19). To ascertain whether continued illumination would maintain elevated levels of P-ERK, lights were left on from CT18 to CT19.5. Under these conditions, P-ERK levels in animals sampled from CT19, but not at CT19.5, were significantly higher than prepulse values and also higher than values obtained after a 30 min light pulse $(p<0.05)$ (Fig. $1 C)$.

A 30 min light pulse presented at CT13-13.5 (during the phase delay portion of the photic PRC) also led to significant increases in P-ERK immunostaining throughout the SCN (see Fig. 6). However, light pulses at CT8, which do not elicit behavioral phase shifts, also did not elicit significant changes in P-ERK levels in the SCN (see Fig. 6). Thus, phosphorylation of ERK in response to a light pulse is phase-gated.

\section{Regulation of P-Elk-1 in the SCN}

When levels of phosphorylated Elk-1 were examined in the SCN in $\mathrm{LD}$ and $\mathrm{DD}$, no significant variation in the number of immunopositive cells (Fig. $2 A, B$ ) or in overall immunostaining (data not shown) was detected. The data presented is from throughout the SCN; however, there was no evidence that P-Elk-1 was rhythmic in any subportion of the SCN (e.g., "core" or "shell" SCN). P-Elk-1 immunostaining was always found to be nuclear in character in the SCN and intergeniculate leaflet (IGL) (Fig. 3A,E), unlike in other CNS regions (hippocampus, cortex) (Fig. 3C,D), where staining was seen in axons/dendrites as well as nuclei. Pretreatment of antisera with P-Elk-1 peptide effectively ablated immunostaining, demonstrating the specificity of the staining described here (Fig. 3B).

On administration of a light pulse at CT18, levels of P-Elk-1 in the SCN were significantly upregulated, with peak levels occurring $1 \mathrm{hr}$ after the onset of the light pulse $(p<0.001$ between 


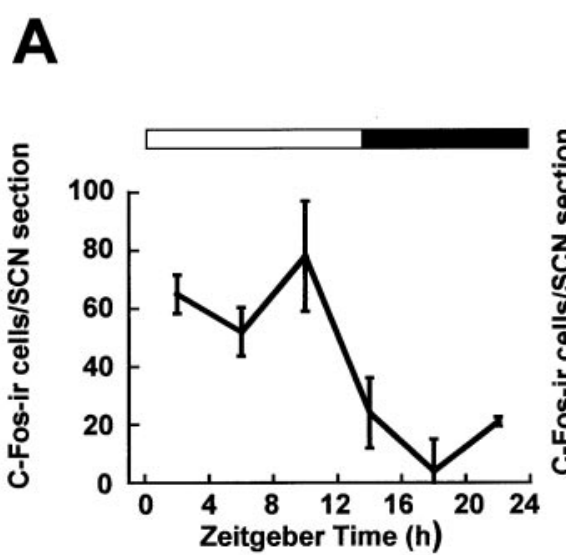

D

CT8

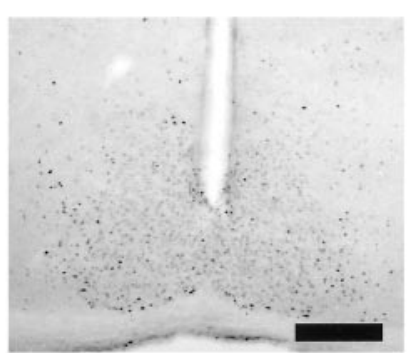

B

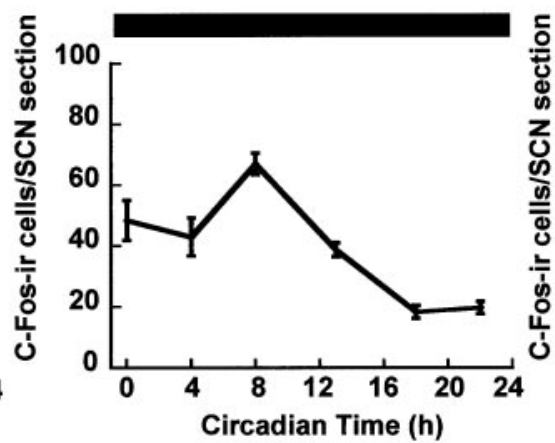

CT18

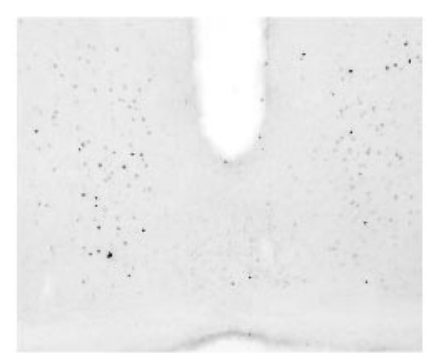

C

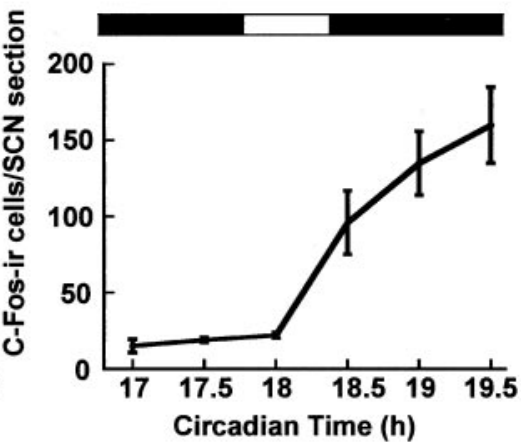

CT18 + Light



Figure 5. Regulation of $\mathrm{C}-$ Fos in the hamster SCN. Diurnal $(A)$ and circadian $(B)$ variation of $\mathrm{C}-$ Fos in the hamster SCN. Numbers of $\mathrm{C}$-Fos-ir cells peak during the mid subjective day, and levels are low throughout the subjective night. C, Photic regulation of C-Fos in the hamster SCN. Light pulses led to a large rise in the number of c-Fos-ir cells in the SCN. $n=4-6$ for each point in $A-C$. $D$, Representative photomicrographs of c-Fos-ir in the SCN sampled during the subjective day in DD (CT8), during the subjective night in DD (CT18), and 90 min after the start of the light pulse (CT18 + Light). Scale bar, $50 \mu \mathrm{m}$.

CT17-17.5 and CT19). P-Elk-1 was consistently most strongly upregulated in the core portion of the SCN, with a distribution similar to that of light-induced c-Fos (Figs. $2 D, 5 D$ ). After this peak, levels started to decline toward baseline. Extension of the light pulse from CT18 to CT19.5, as in Figure 1C, did not significantly alter the time course of P-Elk-1 expression from that achieved with a 30 min pulse (data not shown). Light pulses at CT18 did not affect levels of total Elk-1 protein (Fig. $4 \mathrm{~B}$ ). Light pulses at CT13 also led to significant upregulation of P-Elk-1 $(p<0.05)$, whereas a light pulse at CT8 did not lead to any change in levels of P-Elk-1 in the SCN (see Fig. 6). Thus, similar to P-ERK, photic induction of P-Elk-1 appears to be phasedependent.

\section{c-Fos regulation in the $\mathrm{SCN}$}

We examined the regulation of c-Fos expression in the SCN to allow for comparison with P-ERK and P-Elk-1 expression. Levels of c-Fos immunoreactivity showed diurnal and circadian variation, with levels reaching their maximum during the subjective day and having their nadir during the subjective night (Fig. $5 A, B)$. Highest levels of $\mathrm{c}$-Fos expression during the subjective day or light portion of a LD cycle were in the rostral SCN. Photic stimulation led to upregulation of c-Fos levels with a time course similar to that reported previously, with a phase specificity similar to that for P-ERK and P-Elk-1 (Figs. 5C, 6).

\section{MK-801 treatment}

Treatments of animals with the noncompetitive NMDA-receptor channel blocker MK-801 before a light pulse was found to atten- uate significantly the induction of P-ERK $(p<0.005$ MK-801 compared with controls) (Fig. 7 A,B) and P-Elk-1 $(p<0.005$ MK-801 compared with controls) (Fig. $7 C, D$ ) in the SCN. The core P-ERK staining observed during the subjective night in freerunning hamsters was preserved in animals treated with MK-801 and subsequently light pulsed (Fig. 7A). As reported previously (Abe et al., 1991), c-Fos induction was also attenuated (Fig. $7 E, F)$, although there was a kernel of c-Fos-ir cells induced by the light pulse that was impervious to blockade by MK-801 (Fig. 7E).

\section{U0126 microinjection}

Administration of the MEK inhibitor U0126 before application of a light pulse at CT18 significantly attenuated light-induced phosphorylation of Elk-1 and ERK (Fig. 8A,B). Treatment with U0126, however, did not alter the light-induced expression of c-Fos (Fig. 8C), nor did vehicle DMSO alter the expression of any of the factors examined. U0126 was effective at the amounts of 2.5 and $5 \mathrm{nmol}$, but not $1 \mathrm{nmol}$, when administered to the third ventricle.

\section{Behavioral phase shifts}

Light pulses at CT18 in animals microinjected intracerebroventricularly with vehicle led to a significant phase advance of wheelrunning behavior (125 $\pm 15 \mathrm{~min}$ ) (Fig. 9). Treatment with U0126 $(5 \mathrm{nmol}$ in $1 \mu \mathrm{l}$ ) $30 \mathrm{~min}$ before the light pulse significantly attenuated the phase advance $(43.8 \pm 14.5 \mathrm{~min} ; p<0.01$ compared with vehicle-treated controls) (Fig. 9). 


\section{Light Pulse CT8 Light Pulse CT13}
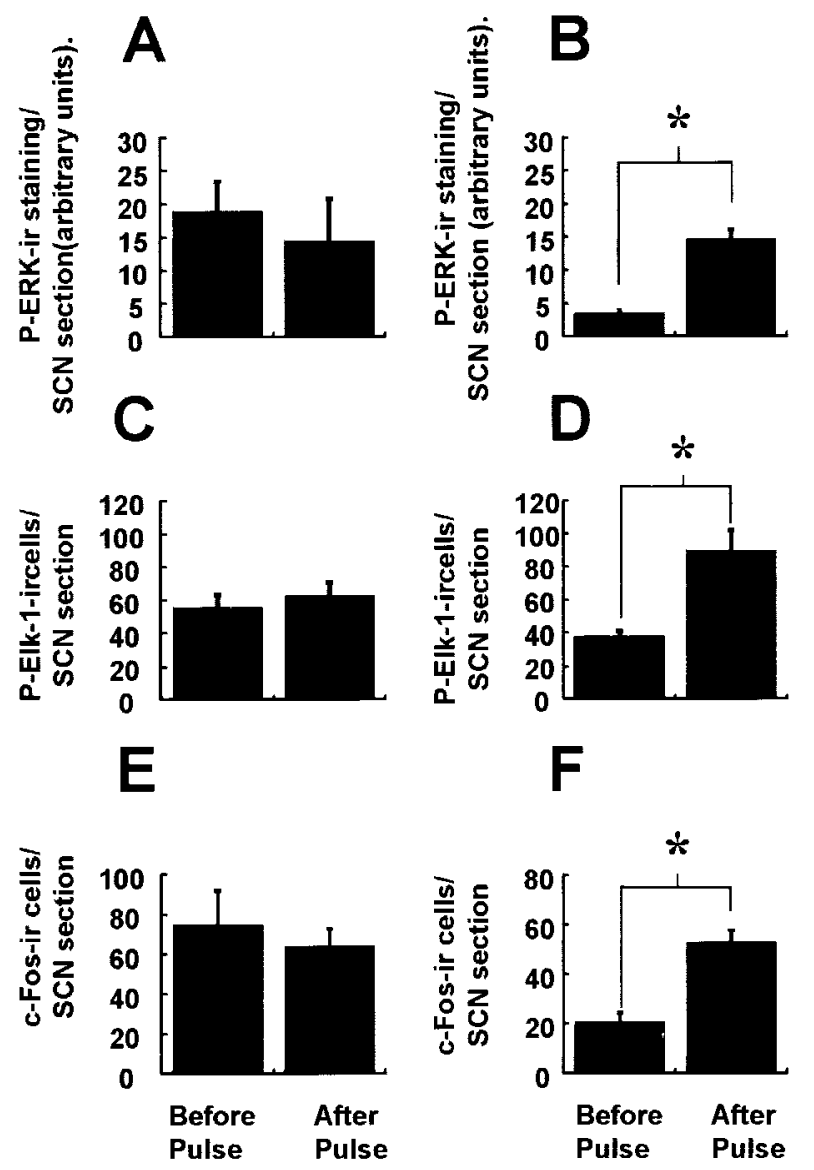

Figure 6. Phase specificity of photic induction of P-ERK, P-Elk-1, and C-Fos in the hamster SCN. Similar to a light pulse at $\mathrm{CT} 18$, a light pulse presented at $\mathrm{CT} 13-13.5$ (which leads to behavioral phase delays) upregulates P-ERK, P-Elk-1, and C-Fos in the hamster SCN $(B, D, F)$. However, light pulse presented at CT8 - CT8.5, which does not lead to behavioral phase shifts, also does not significantly alter levels of P-ERK, P-Elk-1, or C-Fos $(A, C, D) . n=4$ for both the prepulse and light-pulsed groups. ${ }^{*} p<0.01$.

\section{Discussion}

The results presented here illustrate, for the first time, the circadian and photic regulation of the ERK/Elk-1 signaling cascade in the hamster circadian system. These data therefore add considerably to our understanding of the biochemical basis underlying circadian phase-resetting.

Signaling via ERK cascades has been implicated in numerous cellular and physiological processes in the CNS, such as synaptic plasticity and its various behavioral correlates (for review, see Adams and Sweatt, 2002). In recent years attention has focused on what roles the ERK cascade plays in the mammalian circadian system. For example, putative effectors of the ERK cascade, such as CREB (Ginty et al., 1993; Obrietan et al., 1999; Tischkau et al., 2002), modulate phase-resetting in the circadian clock.

To examine the possible role of ERK in circadian processes, we chose the Syrian hamster as our model organism, because this species is the best-characterized mammal from a chronobiological perspective. Our methods allowed us to examine phosphorylated forms of ERK, which are the catalytically active configurations of these enzymes (Sweatt, 2001). P-ERK expression was high during the subjective day in LD or DD, with levels of P-ERK at their nadir during the subjective night. This pattern resembled that reported in this study and previously for c-Fos (Guido et al., 1999), the clock genes per1 and per2 (Tei et al., 1997; Takumi et al., 1998), and AVP (Jin et al., 1999).

Interestingly, there also seemed to be a circadian variation in the distribution of P-ERK in the SCN. During the subjective day, P-ERK was found throughout the rostrocaudal extent of the SCN, in what appear to be axonal and dendritic processes, as well as in perikarya and nuclei. During the subjective night, P-ERK was localized to a cluster of cells in the mid-caudal level of the SCN (Fig. 1D). On initial inspection it would appear that the $\mathrm{P}-\mathrm{ERK}$ cells during the day phase are shell neurons (Hamada et al., 2001) of the SCN, closely resembling the distribution of AVPsynthesizing neurons, whereas the nighttime P-ERK cells closely resemble calbindin-containing cells (Silver et al., 1996). However, a recent study has reported that although the distribution of daytime and nighttime P-ERK cells strongly resembles that of AVP and calbindin, respectively, they do not represent identical populations of cells in that there is no overlap between daytime P-ERK and AVP cells and nighttime P-ERK and calbindin cells (Lee et al., 2003). Thus, the neurochemical phenotype of cells expressing P-ERK in the SCN remains to be ascertained, as does the functional significance of the pattern of distribution. Intriguingly, the core of P-ERK cells is preserved in the SCN of hamsters treated with MK-801 and then subsequently light pulsed at CT18 (Fig. $8 \mathrm{~A}$ ). An interpretation of this, given the findings of Lee et al. (2003) that P-ERK immunoreactivity in the core SCN is ablated after enucleation, is that endogenous P-ERK expression during the subjective night is dependent on nonglutamatergic signals from retinally derived afferents [e.g., pituitary adenylate cyclase activating polypeptide (PACAP)].

Photic induction of P-ERK in the SCN appears to mimic the phase-specific pattern of phase-shifting effects of light in vivo (Daan and Pittendrigh, 1976) and glutamate in vitro (Ding et al., 1994). The pattern of photic-induced P-ERK in the hamster SCN also appears to resemble that reported in the mouse SCN (Obrietan et al., 1998; Butcher et al., 2002). We also report the time course of this photic induction of P-ERK in the SCN: light rapidly, but transiently, induced the phosphorylation of ERK. When the lights were left on after the end of the initial $30 \mathrm{~min}$ light pulse, levels of P-ERK remained high in the SCN. Thus, phosphorylation of ERK appears to be an acute response to light, and on termination of the pulse there is a rapid, presumably phosphatase-mediated dephosphorylation event. It is notable that even when the lights were left on, levels of P-ERK started to decline toward baseline at CT19.5. A possible explanation for this is an autoregulatory mechanism by which activation of the ERK cascade leads to expression of phosphatases that in turn switch off the cascade. A precedent for such a mechanism is found in the hippocampus, where induction of LTP leads to phosphorylation of ERK but also induces the MAP kinase phosphatase (MKP)-1, which in turn returns levels of P-ERK to baseline (Davis et al., 2000). In the chick pineal, where P-ERK shows a circadian rhythm in anti-phase to that reported for the SCN here, a light pulse leads to the dephosphorylation of P-ERK (Sanada et al., 2000), indicative of a vital role for phosphatases.

Were the ERK cascade to be involved in resetting the core molecular clockworks, then it should impinge on gene expression. We chose to examine the role of Elk-1, which has been shown in numerous cell systems to be a putative downstream effector of ERK activation (Yordy and Muise-Helmericks, 2000). When Elk-1 is phosphorylated it binds to its consensus sequence (CAGGA) in conjunction with a dimer of the serum response 
A
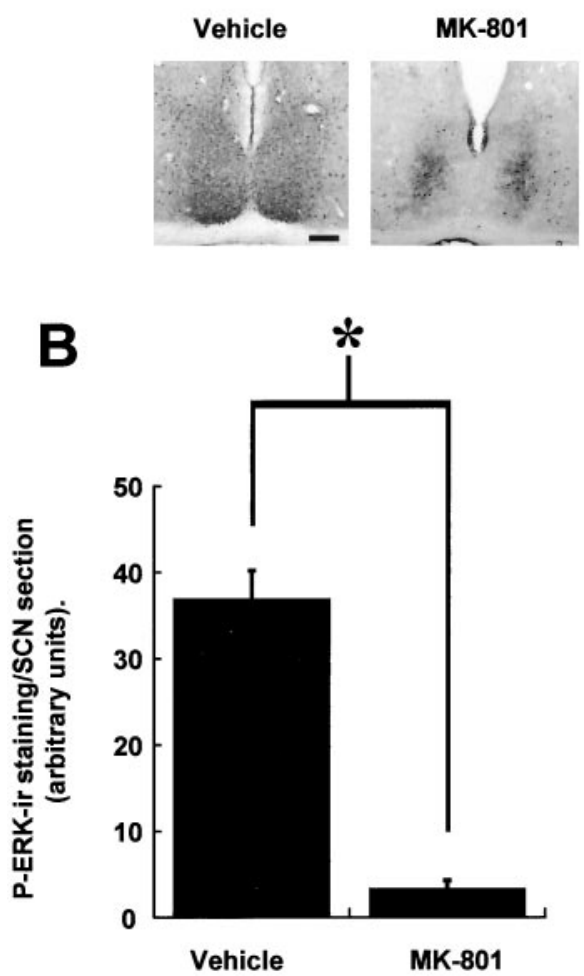

C
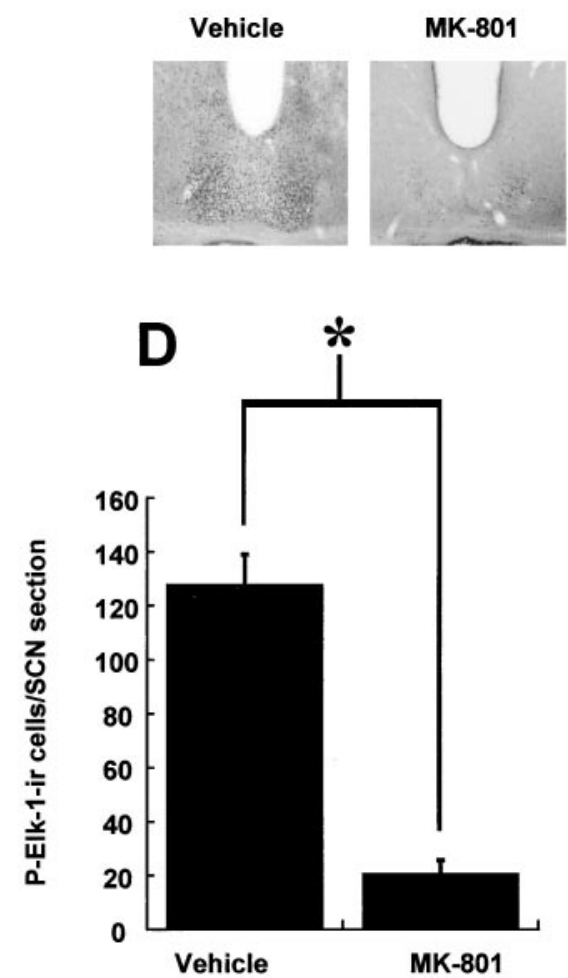

E
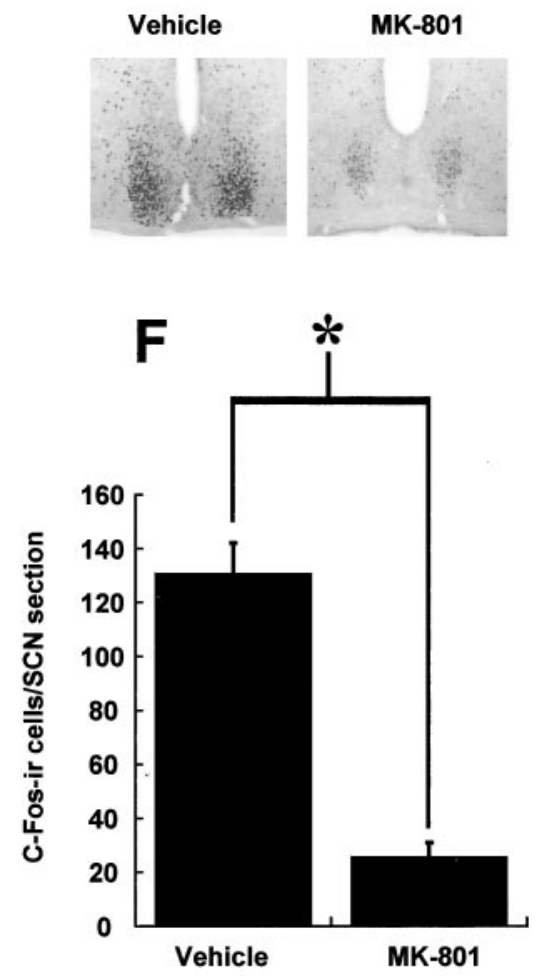

Figure 7. The NMDA-receptor channel blocker MK-801 attenuates photic induction of P-ERK $(A, B), P-E l k-1(C, D)$, and $c$-Fos $(E, F)$ in the SCN. $n=4$ for vehicle controls and MK-801 treatments. Representative photomicrographs of sections from CT18.5 (A), CT19 (C), and CT19.2 (E) after a light pulse at CT18. * $p<0.01$. Scale bar, $50 \mu \mathrm{m}$.

factor on SREs present on gene promoter sequences (Wasylyk et al., 1998). Thus, phosphorylation of Elk-1 is a vital event in mediating SRE-driven transcription.

SREs are present in a number of photically regulated genes [e.g., c-fos (Rusak et al., 1990), per1 (Takumi et al., 1998; Wilsbacher et al., 2002), zif268 (Dong et al., 2002)]. Therefore, we wished to examine the possibility that Elk-1 is involved in mediating photic phase shifting in the hamster. Unlike P-ERK, P-Elk-1 was not found to display significant variation under diurnal or free-running conditions. However, after administration of a light pulse at CT18, levels of P-Elk-1 were strongly upregulated. The time course of P-Elk-1 was somewhat slower than that found for P-ERK, probably because of the fact that in the SCN, P-Elk-1 was found to be solely nuclear, and its phosphorylation may require a cytoplasm-to-nucleus translocation event, with the relative delay associated with such mechanisms (Martin et al., 1997). The induction of P-Elk-1 by light also appears to be phase-gated in a similar manner to P-ERK.

Given that total levels of Elk-1 (and ERK) do not vary under any of the conditions examined, we can conclude that the changes in P-Elk-1 observed are functions of phosphorylation/dephosphorylation events, and not caused by changes in overall expression levels. Therefore, Elk-1 may represent a downstream target of the ERK cascade in relation to photic resetting, but not seemingly in the free-running clock. One possible mechanism, which may underpin this difference, is subcellular availability of substrate; under free-running conditions components of the ERK cascade may be held by scaffold proteins in cellular compartments where Elk-1 is not available as a substrate (Van Droogen and Peter, 2002). Another possibility is that phosphorylation of Elk-1 is under the control of signaling pathways other than ERK; these additional pathways may be activated by light but do not cycle under free-running conditions. Elk-1 is also a putative target for other MAP kinase pathways, such as the p38 MAP kinase and C-terminal-Jun kinase (Wasylyk et al., 1998), and the role of these kinases in circadian processes has yet to be examined.

Our finding that the MEK inhibitor U0126 attenuated photicinduced phosphorylation of both ERK and Elk-1 indicates that Elk-1 is indeed a downstream component of the ERK cascade in the SCN. Also, because U0126 attenuates light-induced phase shifts in behavioral rhythms, both in our study and in a study in the mouse (Butcher et al., 2002) [however, see Yokota et al. (2001)], further weight may be added to the argument that Elk-1 is involved in the biochemical mechanism of photic resetting of circadian phase. Phosphorylation of both ERK and Elk-1 in the SCN, similar to behavioral phase shifting (Rea et al., 1993), in response to photic stimulation is dependent on glutamatergic transmission via the NMDA receptor, because pretreatment with MK-801 attenuates P-ERK and P-Elk-1 induction.

Transcription of the immediate early gene $c$-fos has been reported to be under control of SREs on its promoter in neuronal systems (Vanhoutte et al., 1999). Thus, phosphorylation of Elk-1 in the SCN may be an event in the sequence that leads to expression of c-Fos in the SCN in response to a light pulse (Rusak et al., 1990). However, inhibition of the ERK cascade by U0126 failed to attenuate c-Fos induction. Therefore, either ERK/Elk-1 is not involved in c-Fos induction in the SCN or the $c$-fos promoter is under redundant control that allows for sufficient compensation after ablation of ERK/Elk-1 signaling. Such dissociation between behavioral phase shifts and $c$-fos induction is found in a study on mice lacking the PACAP type I receptor (Hannibal et al., 2001) that exhibit normal photic $c$-fos induction but manifest phase 


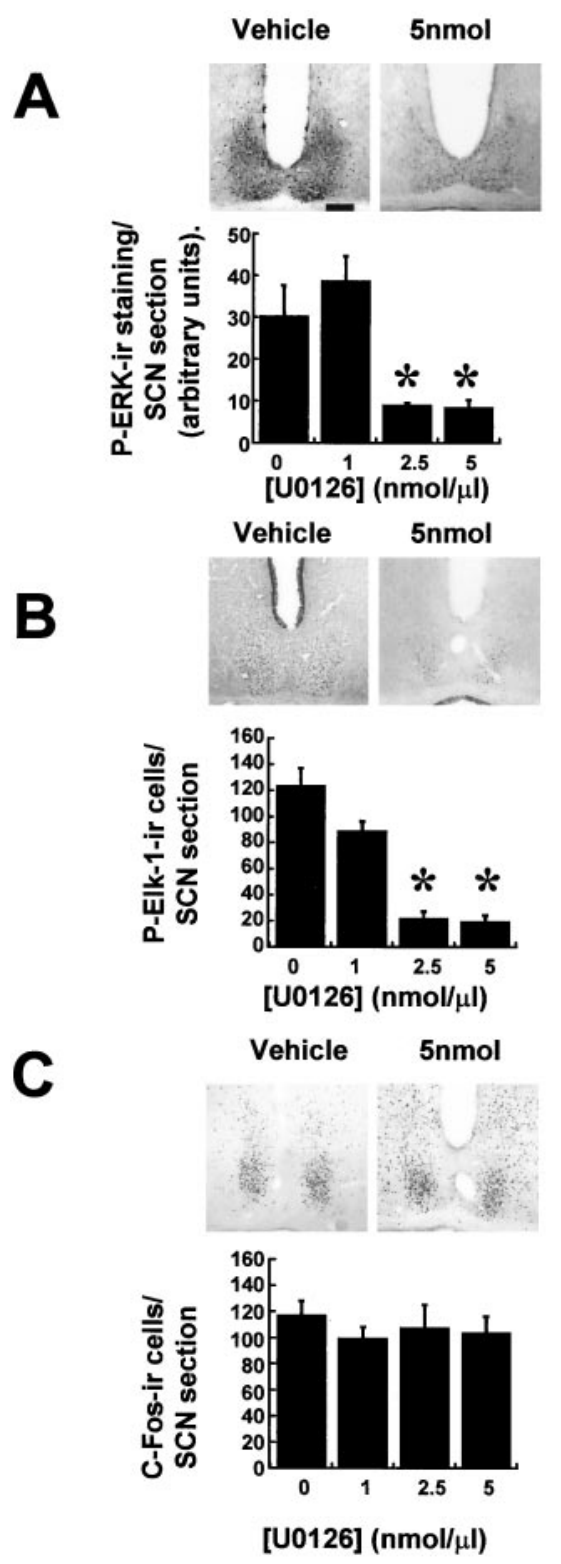

Figure 8. The ERK cascade inhibitor U0126 attenuates photically induced P-ERK and P-Elk-1 but not c-Fos in the SCN. Microinjection of $2.5 \mathrm{nmol}(n=5)$ or $5 \mathrm{nmol}(n=6)$ of U0126 $(1 \mu \mathrm{l})$ into the third ventricle before application of a light pulse led to significant attenuation of $P$-ERK and P-Elk-1 induction $(A, B)$ but did not attenuate c-Fos induction $(C)$. Vehicle DMSO $(1 \mu \mathrm{l} /$ $10 \% ; n=6)$ or $1 \mathrm{nmol}$ of $\mathrm{U} 0126(n=4)$ did not have any significant effect. ${ }^{*} p<0.01$. Scale bar, $50 \mathrm{~mm}$.

delays to late-night light pulses instead of the expected phase advances. Thus, c-Fos induction may not be vital in driving behavioral phase-shifting.

The data presented uniquely illustrate that the ERK/Elk-1 cascade is an integral component of the phase-resetting mechanism in the mammalian circadian clock. Our findings, taken in conjunction with recently published work (Butcher et al., 2002; Lee et al.,2003), provide a fascinating insight into the complex nature of intracellular regulation of time-keeping processes.

\section{References}

Abe H, Rusak B, Robertson HA (1991) Photic induction of Fos protein in the suprachiasmatic nucleus is inhibited by the NMDA receptor antagonist MK-801. Neurosci Lett 127:9-12.
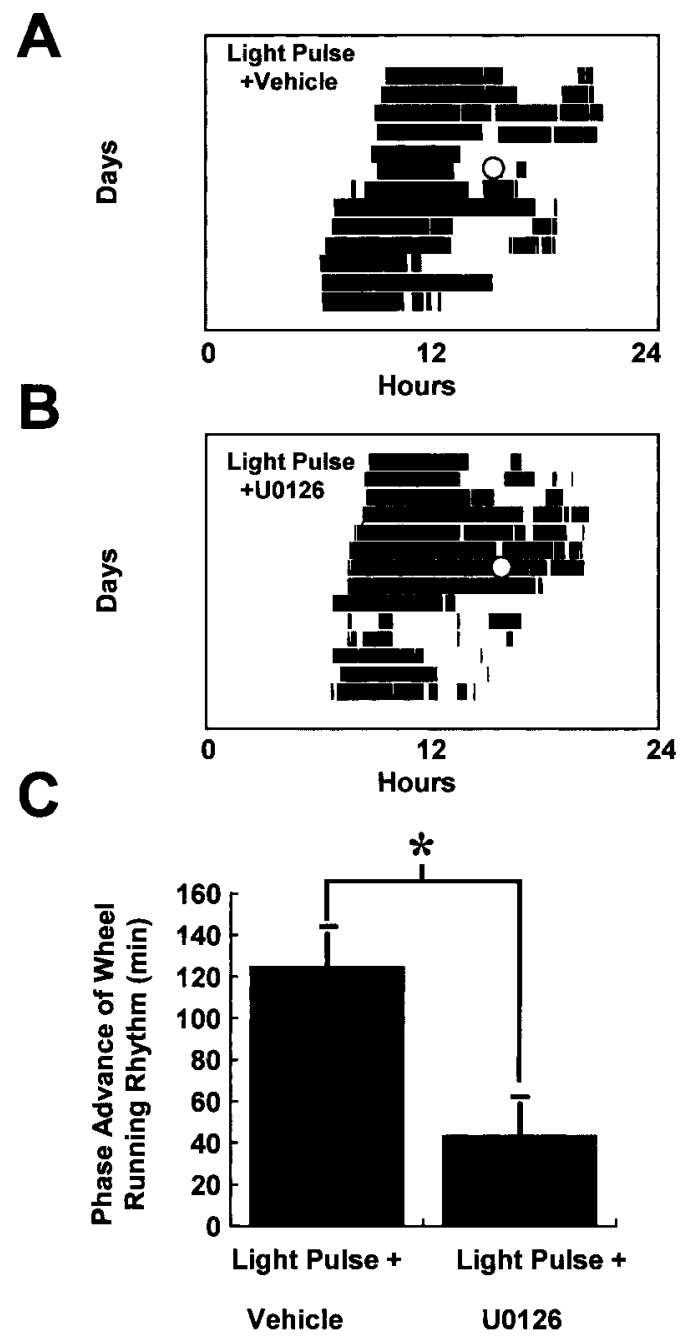

Figure 9. The ERK cascade inhibitor U0126 attenuates photically induced phase shifts of behavioral rhythms. Microinjection of U0126 ( $5 \mathrm{nmol}$ in $1 \mu \mathrm{l} ; n=6)(A) 30 \mathrm{~min}$ before a light pulse (CT18-18.5) significantly decreased the magnitude of phase advance compared with vehicle-treated controls $(n=5 ; B, C)$. In $A$ and $B$, the light-filled circle denotes application of the light pulse, and in $C^{*} p<0.01$.

Adams JP, Sweatt JD (2002) Molecular psychology: roles for the ERK MAP kinase cascade in memory. Annu Rev Pharmacol Toxicol 42:135-163.

Allada R, Emery P, Takahashi JS, Rosbash M (2001) Stopping time: the genetics of fly and mouse circadian clocks. Annu Rev Neurosci 24:1091-1119.

Arima H, House SB, Gainer H, Aguilera G (2002) Neuronal activity is required for the circadian rhythm of vasopressin gene transcription in the suprachiasmatic nucleus in vitro. Endocrinology 143:4165-4171.

Butcher G, Dziema H, Collamore M, Burgoon PW, Obrietan K (2002) The p42/44 MAP kinase pathway couples photic input to circadian clock entrainment. J Biol Chem 277:29519-29525.

Coogan AN, O’Leary DM, O'Connor JJ (1999) p42/44 MAP kinase inhibitor PD98059 attenuates multiple forms of synaptic plasticity in the rat dentate gyrus in vitro. J Neurophysiol 81:103-110.

Daan S, Pittendrigh CS (1976) A functional analysis of circadian pacemakers in rodents: the variability of phase-response curves. J Comp Physiol [A] 106:255-266.

Davis S, Vanhoutte P, Pages C, Caboche J, Laroche S (2000) The MAPK/ ERK cascade targets both Elk-1 and cAMP response element-binding protein to control long-term potentiation-dependent gene expression in the dentate gyrus in vivo. J Neurosci 20:4563-4572.

Ding JM, Chen D, Webber ET, Fairman LE, Rea MA, Gillette MU (1994) Resetting the biological clock: mediation of nocturnal circadian shifts by glutamate and NO. Science 266:1713-1717. 
Dong YN, Goguen D, Robertson HA, Rusak B (2002) Anatomical and temporal differences in the regulation of ZIF268 (NGFI-A) protein in the hamster and mouse suprachiasmatic nucleus. Neuroscience 111:567-574.

Ebling FJP (1996) The role of glutamate in the photic regulation of the suprachiasmatic nucleus. Prog Neurobiol 50:109-131.

Ginty DD, Kornhauser JM, Thompson MA, Bading H, Mayo KE, Takahashi JS, Greenberg ME (1993) Regulation of CREB phosphorylation in the suprachiasmatic nucleus by light and a circadian clock. Science 260:238-241.

Guido ME, Gougen D, deGuido L, Robertson HA, Rusak B (1999) Circadian and photic regulation of immediate early gene expression in the hamster suprachiasmatic nucleus. Neuroscience 90:555-571.

Hamada T, LeSauter J, Venuti JM, Silver R (2001) Expression of period genes: rhythmic and nonrhythmic components of the suprachiasmatic nucleus pacemaker. J Neurosci 21:7742-7750.

Hannibal J, Jamen F, Nielsen HS, Journot L, Brabet P, Fahrenkrug J (2001) Dissociation between light-induced phase shift of the circadian rhythm and clock gene expression in mice lacking the pituitary adenylate cyclase activating polypeptide type 1 receptor. J Neurosci 21:4883-4890.

Hayashi Y, Sanada K, Fukuda Y (2001) Circadian and photic regulation of MAP kinase by Ras- and protein phosphatase-dependent pathways in the chick pineal gland. FEBS Lett 491:71-75.

Jin XW, Shearmann LP, Weaver DR, Zylka MJ, DeVries GJ, Reppert SM (1999) A molecular mechanism regulating rhythmic output from the suprachiasmatic circadian clock. Cell 96:57-68.

Ko GY-P, Ko ML, Dryer SE (2001) Circadian regulation of cGMP-gated cationic channels of chick retinal cones: ERK MAP kinase and $\mathrm{Ca}^{2+}$ / calmodulin-dependent protein kinase II. Neuron 29:255-266.

Lee C, Etchegaray J-P, Cagampang FR, Loudon ASI, Reppert SM (2001) Posttranslational mechanisms regulate the mammalian circadian clock. Cell 107:855-867.

Lee HS, Nelms JL, Nguyen M, Silver R, Lehman MN (2003) The eye is necessary for a circadian rhythm in the suprachiasmatic nucleus. Nat Neurosci 6:111-112.

Lowrey PL, Shimomura K, Antoch MP, Yamazaki S, Zemenides PD, Ralph MR, Menaker M, Takahashi JS (2000) Positional syntenic cloning and functional characterization of the mammalian circadian mutation tau. Science 288:483-492.

Martin KC, Michael D, Rose JC, Barad M, Casadio A, Zhu H, Kandel ER (1997) MAP kinase translocates into the nucleus of the presynaptic cell and is required for long-term facilitation in Aplysia. Neuron 18:898-912.

Moore RY, Lenn NJ (1972) A retinohypothalamic projection in the rat. J Comp Neurol 146:1-14.

Obrietan K, Impey S, Storm D (1998) Light and circadian rhythmicity regulate MAP kinase activation in the suprachiasmatic nuclei. Nat Neurosci 1:693-700.

Obrietan K, Impey S, Smith D, Athos J, Storm DR (1999) Circadian regulation of cAMP response element-mediated gene expression in the suprachiasmatic nuclei. J Biol Chem 274:17748-17755.

Oh-Hashi K, Naruse Y, Tanaka M (2002) Intracellular calcium mobilization induces period genes via a MAP kinase pathway in NIH3T3 cells. FEBS Lett 516:101-105.

Ralph MR, Foster RG, Davis FC, Menaker M (1990) Transplanted suprachiasmatic nucleus determines circadian phase. Science 247:975-979.
Rea MA, Buckley B, Lutton LM (1993) Local administration of EAA antagonists blocks light-induced phase shifts and c-Fos expression in the hamster SCN. Am J Physiol 265:R1191-1198.

Reppert SM, Weaver DR (2002) Coordination of circadian timing in mammals. Nature 418:935-941.

Rusak B, Robertson HA, Wisden W, Hunt SP (1990) Light pulses that shift rhythms induce gene expression in the suprachiasmatic nucleus. Science 248:1237-1241.

Sanada K, Hayashi Y, Harada Y, Okano T, Fukuda Y (2000) Role of circadian activation of mitogen-activation protein kinase in chick pineal clock oscillation. J Neurosci 20:986-991.

Sanada K, Okano T, Fukuda Y (2002) Mitogen-activated protein kinase phosphorylates and negatively regulates basic helix-loop-helix-PAS transcription factor BMAL1. J Biol Chem 277:267-271.

Silver R, Romero MT, Besner HR, Leak R, Nunez JM, LeSauter J (1996) Calbindin D-28k cells in the hamster SCN express light-induced Fos. NeuroReport 7:1224-1228.

Sweatt JD (2001) The neuronal MAP kinase cascade: a biochemical signal integration system subserving synaptic plasticity and memory. J Neurochem 76:1-10.

Takumi T, Matsubara C, Shigeoyoshi Y, Taguchi K, Yagita K, Maebayashi Y, Sakakida Y, Okamura K, Takashima K, Okamura H (1998) A new mammalian period gene predominantly expressed in the suprachiasmatic nucleus. Genes Cells 3:167-176.

Tei H, Okamura H, Shigeyoshi Y, Fukuhara YC, Ozawa R, Hirose Y, Sakaki Y (1997) Circadian oscillations of a mammalian homologue of the Drosophila period gene. Nature 389:515-518.

Tischkau SA, Mitchell JW, Tyan S-H, Buchanan GF, Gillette MU (2002) CREB-dependent activation of Per1 is required for light-induced signaling in the suprachiasmatic nucleus circadian clock. J Biol Chem 278:718-723.

Van Droogen F, Peter M (2002) MAP kinase cascades: scaffolding signal specificity. Curr Biol 12:53-55.

Vanhoutte P, Barnier J-V, Guibert B, Pages C, Besson M-J, Hipskind RA, Caboche J (1999) Glutamate induces phosphorylation of Elk-1 and CREB, along with c-fos activation, via an extracellular signal-regulated kinase-dependent pathway in brain slices. Mol Cell Biol 19:136-146.

Wasylyk B, Hagman J, Guiterrez-Hartmann A (1998) Ets transcription factors: nuclear effectors of the Ras-MAP kinase signaling pathway. Trends Biochem Sci 23:213-216.

Wilsbacher LD, Yamazaki S, Herzog ED, Song E-J, Radcliffe LA, Abe M, Block G, Spitznagel E, Menaker M, Takahashi JS (2002) Photic and circadian expression of luciferase in mperiod1-luc transgenic mice in vivo. Proc Natl Acad Sci USA 99:489-494.

Yokota S-I, Yamamoto M, Moriya T, Akiyama M, Fukunaga K, Miyamoto E, Shibata S (2001) Involvement of calcium-calmodulin protein kinase but not mitogen-activated protein kinase in light-induced phase-delays and per gene expression in the suprachiasmatic nucleus of the hamster. J Neurochem 77:618-627.

Yordy JS, Muise-Helmericks RC (2000) Signal transduction and the Ets family of transcription factors. Oncogene 19:6503-6513. 